\title{
Tecnologia de informação e comunicação no ensino de enfermagem
}

Information and communication technology in nursing education Tecnologías de la información y la comunicación en la enseñanza de enfermería

Angela Gilda Alves ${ }^{1}$ ito https://oridid.org/0000-0001-8709-8933 Flaviane Cristina Rocha Cesar ${ }^{1}$ ic https://orcid.org/0000-0002-2659-2871

Cleusa Alves Martins' ${ }^{1}$ ib https://orcid.org/0000-0002-7210-6934

Luana Cássia Miranda Ribeiro ${ }^{1}$ io hitps://orcid.orgy/0000-0002-4254-2030 Lizete Malagoni de Almeida Cavalcante Oliveira ${ }^{1}$ io hitps://orid.org/0000-0002-1055-1354

Maria Alves Barbosa ${ }^{1}$ id https://orcid.org/0000-0002-0861-9655 Katarinne Lima Moraes ${ }^{1}$ id https://orcid.org/0000-0001-6169-0461

Como citar: Alves AG, Cesar FC, Martins CA, Ribeiro LC, Oliveira LM, Barbosa MA, et al. Information and communication technology in nursing education. Acta Paul Enferm. 2020;33:eAPE20190138.

DOI

http://dx.doi.org/10.37689/actaape/2020A001385

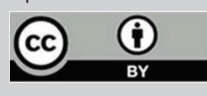

Descritores

Tecnologia da informação; Ensino; Educação em enfermagem; Pesquisa em educação de enfermagem

Keywords Information technology: Teaching; Education, nursing; Nursing education research

Descriptores

Tecnología de la información; Enseñanza; Educación en enfermería; Investigación en educación de enfermería

Submetido 2 de Junho de 2019

Aceito

18 de Fevereiro de 2020

\section{Autor correspondente}

Angela Gilda Alves

E-mail: angelagildaalves@gmail.com

\section{Resumo}

Objetivo: Analisar a utilização de Tecnologias de Informação e Comunicação (TIC) no processo de ensino e aprendizado por docentes de enfermagem.

Métodos: Estudo qualitativo com abordagem na Teoria Fundamentada nos Dados Straussiana, realizado com professores enfermeiros em faculdades de enfermagem públicas e privadas do Centro-Oeste brasileiro. Para análise dos dados realizou-se codificação aberta - identificação de códigos; codificação axial - determinar as subcategorias e categorias; codificação seletiva - para constituir uma categoria central que representasse 0 conjunto de dados analisados.

Resultados: A codificação aberta realizada com a resposta dos 22 professores de enfermagem, identificou os códigos: aprendizagem, clínicas, associação, educação permanente, didática, WhatsApp, Facebook e Youtube. A codificação axial resultou em categorias (fragilidade da formação docente; utilização de mídias sociais como procedimento de ensino) e subcategorias (conhecimento; qualificação permanente; ferramentas) que representam o fenômeno estudado. A codificação seletiva refinou todas as categorias e subcategorias emergindo a categoria central nominada " 0 desafio de envolver 0 aluno no processo de ensino aprendizagem e a distância entre gerações professor-aluno".

Conclusão: Foi possível identificar o uso das TIC por meio das redes sociais (YouTube; Facebook e WhatsApp) como estratégias tecnológicas positivas no ensino de ensino aprendizado da enfermagem. No entanto, sua implementação ainda precisa transpor desafios relacionados com prática pedagógica, condições de acesso dos estudantes à tecnologia e conflito de gerações. Essas dificuldades apontam para a necessidade de discutir a formação do enfermeiro educador e a organização acadêmica em consonância com o perfil discente contemporâneo, cada vez mais conectado às tecnologias.

\section{Abstract}

Objective: To analyze the use of Information and Communication Technologies (ICTs) by nursing professors in the teaching and learning process.

Method: Qualitative study with an approach based on the Straussian Grounded Theory, conducted with nursing professors at public and private nursing schools in the Mid-west Region of Brazil. For data analysis, the steps were open coding - identification of codes; axial coding - determining subcategories and categories; selective coding - identifying a central category that represented the set of data analyzed.

Results: The open coding carried out with the answers from the 22 nursing professors, identified the codes: learning, clinic, association, continuing education, didactic, WhatsApp, Facebook and Youtube. The axial coding resulted in categories (fragility of lecturer training; use of social networks as a teaching method) 
and subcategories (knowledge; continuing qualification; tools) which represent the studied phenomenon. The selected coding refined all categories and subcategories, giving rise to the central category called "The challenge of involving the student in the teaching-learning process and the generation gap between lecturer and students".

Conclusion: It was possible to identify the use of ICTs through social networks (YouTube; Facebook and WhatsApp) as positive technological teaching strategies in nursing learning. However, for their implementation, it is necessary to overcome challenges related to pedagogical practice, students' access to technology and generational conflicts. These difficulties point to the need to discuss the education of the nurse lecturer and an academic organization aligned with the contemporary profile of the students, who are increasingly connected to technologies.

\section{Resumen}

Objetivo: Analizar la utilización de tecnologías de la información y la comunicación (TIC) en el proceso de enseñanza y aprendizaje por parte de docentes de enfermería.

Métodos: Estudio cualitativo con enfoque en la teoría fundamentada en los datos de Strauss, realizado con profesores enfermeros en facultades de enfermería públicas y privadas de la región Centro-Oeste de Brasil. Para analizar los datos se realizó codificación abierta (identificación de códigos); codificación axial (determinar las subcategorías y categorías); codificación selectiva (para elaborar una categoría central que representara el conjunto de datos analizados).

Resultados: La codificación abierta realizada con la respuesta de los 22 profesores de enfermería identificó los códigos: aprendizaje, clínicas, asociación, educación permanente, didáctica, WhatsApp, Facebook y YouTube. La codificación axial dio como resultado categorías (fragilidad de la formación docente, utilización de medios de comunicación sociales como procedimiento de enseñanza) y subcategorías (conocimiento, cualificación permanente, herramientas) que representan el fenómeno estudiado. La codificación selectiva refinó todas las categorías y subcategorías y surgió la categoría central denominada "El desafío de involucrar al alumno en el proceso de enseñanza-aprendizaje y la distancia entre generaciones profesor-alumno".

Conclusión: Fue posible identificar el uso de las TIC por medio de las redes sociales (YouTube, Facebook y WhatsApp) como estrategias tecnológicas positivas en la enseñanza de la enseñanza-aprendizaje de enfermería. Sin embargo, para implementarse, aún es necesario superar desafíos relacionados con la práctica pedagógica, las condiciones de acceso de los estudiantes a la tecnología y el conflicto generacional. Estas dificultades señalan la necesidad de debatir sobre la formación del enfermero educador y la organización académica de acuerdo con el perfil actual de los estudiantes, cada vez más conectados a las tecnologías.

\section{Introdução}

A importância social das tecnologias de informação e comunicação (TIC) requer atualização das práticas de ensino para apoiar conhecimento e avanço da enfermagem. Essas tecnologias compreendem meios técnicos que permitem o compartilhamento de informaçóes e os processos comunicativos por meio de recursos como computadores, internet e mídias sociais. ${ }^{(1)}$

As TIC tornaram-se elemento comum no cotidiano, foram atreladas ao setor da saúde ${ }^{(2)}$ e, nas salas de aula, podem exercer influência significativa no processo de aprendizado. ${ }^{(3,4)}$ Para a enfermagem, as TIC podem apoiar a autonomia no processo de buscar conhecimento, a apreensão de conteúdo, ${ }^{(5,6)}$ a tomada de decisão clínica $^{(2)}$ e a qualidade da prestação de cuidados de enfermagem. ${ }^{(7)}$ Nesse sentido, estima-se que até 2025 a incorporação das TIC seja componente indispensável aos currículos e à prática docente de enfermagem. ${ }^{(8)}$

Em contraponto a tendência de ensino de enfermagem mundial, ${ }^{(8)}$ tem sido relatado que professores de enfermagem apresentam baixa aceitação e restrições a utilização das TIC, provavelmente devido a estrutura física, financeira e técnica das instituiçóes de ensino. ${ }^{(9,10)}$

Os professores exercem papel central na implementação de práticas pedagógicas inovadoras. Apesar disso, revisão sistemática da literatura iden- tificou publicaçóes voltadas apenas ao rastreamento de doenças e experiências dos estudantes com uso de redes sociais para aprendizado, não sendo encontrado estudos sobre a aplicação da TIC na ótica da prática pedagógica do docente. ${ }^{(11)}$

Deve-se considerar que o conhecimento é um instrumento de transformação. Nesse sentido, o empoderamento do sujeito é uma consequência da apropriação do saber e constitui etapa preliminar para a ação dos indivíduos. ${ }^{(12)}$ Esse referencial serve de apoio para a ideia de que o uso das TIC, atualmente requerido na prática clínica de enfermagem, pressupóe adquirir durante a formação conhecimentos e habilidades a seu respeito, sendo o professor o mediador desse processo. ${ }^{(8)}$

A experiência dos professores no uso das TIC pode evidenciar se a dinâmica de sala de aula é favorável ao desenvolvimento de competências tecnológicas nos estudantes de enfermagem. Assim, este estudo objetivou analisar a experiência dos docentes de enfermagem na utilização de TIC no processo ensino e aprendizado.

\section{Métodos}

Estudo qualitativo com abordagem na Teoria Fundamentada nos Dados (TFD) Straussiana. A 
TFD permite tratar de forma interpretativa e sistemática experiências e realidades dos atores. $\mathrm{O}$ modelo paradigmático de verificação dessa teoria compreende análise e organização dos dados para interpretar cada categoria/subcategoria elencada, possibilitando resultados fieis à realidade estudada. ${ }^{(13)}$ Assim, a TFD foi adotada nesta pesquisa para: 1) construção de quadro com categorias e subcategorias, que devem ser consideradas para compreender a dinâmica do uso das TIC no ensino de enfermagem; 2) emergir a categoria central, também denominada de generalização teórica.

Os participantes foram recrutados entre agosto e novembro de 2015 por amostragem intencional das seis instituiçôes de ensino superior (IES) públicas e privadas de uma capital do Centro-Oeste brasileiro. Incluiu-se professores enfermeiros, efetivos de IES reconhecidas pelo Ministério da Educação do Brasil e com mais de cinco anos de funcionamento. Foram excluídos os docentes afastados de suas atividades por qualquer motivo no período da coleta dos dados. Após a amostragem inicial intencional, o recrutamento dos participantes continuou até que a saturação dados fosse alcançada. ${ }^{(13)}$

A operacionalizaçáo da coleta de dados foi conduzida pela primeira autora desse estudo, envolveu visita à coordenação do curso de enfermagem de cada IES e solicitação de autorização para o estudo. Nesse encontro, a coordenação do curso forneceu nome e número de telefone dos docentes que atendiam aos critérios de inclusão e exclusão do estudo. Posteriormente, em contato telefônico, esses professores foram abordados, informados sobre os objetivos da pesquisa e convidados a participar. Os que aceitaram, agendaram o encontro de acordo com suas possibilidades para a entrevista.

Após a leitura e assinatura do Termo de Consentimento Livre e Esclarecido, as entrevistas foram realizadas pela primeira autora do estudo, em encontro único com cada participante, e em ambiente livre de ruídos e interrupções, na própria IES. A entrevista foi conduzida a partir da pergunta norteadora "Qual é sua experiência na utilização das Tecnologias de Informação e Comunicação no contexto pedagógico de suas aulas?”. Após isso, a primeira autora perguntou "tem algo que você gos- taria de acrescentar sobre a sua prática docente e o uso das TIC". Todos os registros foram realizados por meio de gravação digital.

A transcrição de cada entrevista foi realizada pela entrevistadora logo após sua realização.

\section{Análise dos dados}

Ao final das transcriçóes, as entrevistas foram submetidas à análise e a interpretação dos conteúdos, de acordo com a proposta metodológica da Teoria Fundamentada nos Dados, dividida estrategicamente em três níveis. ${ }^{(13)}$

A análise de primeiro nível compreendeu a leitura criteriosa das entrevistas, denominada de codificação aberta, com identificação de códigos (palavras ou expressóes) mais prevalentes nas falas dos participantes. ${ }^{(13)}$ Em seguida, esses códigos foram cadastrados no software Atlas/ti.

A análise de segundo nível, denominada codificação axial, contemplou o agrupamento dos códigos por semelhança e/ou proximidade, originando subcategorias que reuniam núcleos de sentido comuns. Posteriormente, o agrupamento dessas subcategorias sustentou a construção de categorias. ${ }^{(13)}$

Por fim, no terceiro nível de análise, definido como codificação seletiva, foi realizado o refinamento, integração e inter-relação das categorias para constituir uma categoria central que representasse o conjunto de dados analisados. Essa análise pressupóe a identificação indutiva e hierarquização das categorias com base nos objetivos da pesquisa, resultando em um eixo temático ou teoria emergente, cuja validade é confirmada e validada por meio da revisita ao banco de dados para verificação da representatividade da teoria em relação aos dados coletados. ${ }^{(13)}$ A análise e interpretação dos conteúdos transcritos são ilustrados na figura $1 .^{(13)}$

\section{Interpretação dos dados}

A interpretação dos dados possui importante papel na TFD como um processo sistemático. ${ }^{(14)}$ A primeira autora, que conduziu todas as entrevistas e principalmente interpretou os dados, é formada em enfermagem, gestão de grupos, é docente de enfermagem e trabalha como coordenadora de curso de enfermagem há 10 anos no Centro-Oeste brasileiro. Além disso, a primei- 


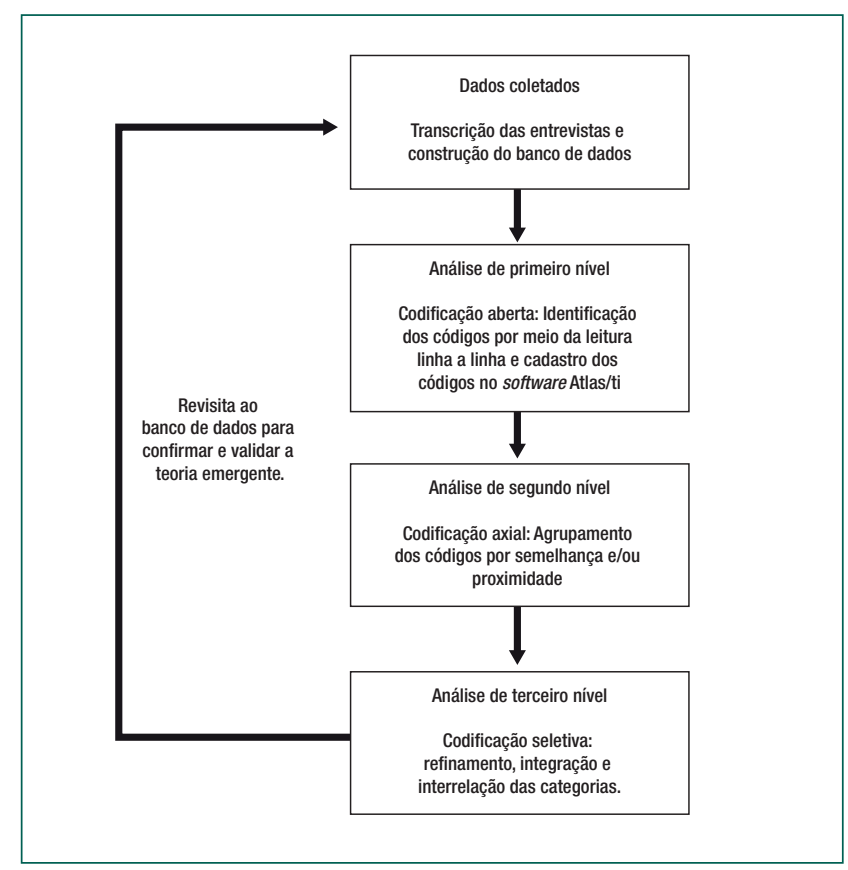

Figura 1. Fluxograma da análise e interpretação das entrevistas de docentes de enfermagem sobre 0 uso pedagógico de tecnologias de informação e comunicação baseada na Teoria Fundamentada nos Dados Straussiana

ra autora tem estudado TFD e prática pedagógica há um longo período, tendo defendido uma dissertação de mestrado e produzido artigos nessa temática. Todos os demais autores possuem experiência em análise de dados qualitativos e compartilham do ponto de vista epistemológico Straussiano. Essas experiências refletiram na análise de dados desta pesquisa.

\section{Aspectos éticos}

Este estudo seguiu as diretrizes para pesquisa em seres humanos vigentes no Brasil, recolhendo o consentimento livre e esclarecido de todos os participantes, tendo obtido aprovação em Comitê de Ética em Pesquisa n 1.314 .801$.

\section{Resultados}

Participaram do estudo 22 professores, predominando mulheres $(19-86 \%)$, idade superior a 45 anos $(12-46 \%)$, mais de 15 anos de profissão (11 - 50\%), título de Mestre (9 - 42\%), vinculados à rede privada de ensino $(18-82 \%)$ e com regime de trabalho de 20 horas semanais (16-74\%).
A codificação aberta relevou os códigos: aprendizagem, clínicas, associação, educação permanente, didática, WhatsApp, Facebook e Youtube. A codificação axial resultou em categorias (Fragilidade da formação docente; Utilização de mídias sociais como procedimento de ensino) e subcategorias (Conhecimento; Qualificação permanente; Ferramentas) que representam o fenômeno estudado. Nesse sentido, foi possível inferir que na percepção dos docentes entrevistados, o conhecimento foi considerado a base de sustentação para o uso de TIC (Facebook, You tube, Whatsapp).

Destaca-se que os docentes associaram as TIC com o aprendizado dos estudantes e reconheceram a educação permanente como propulsora da melhoria das práticas pedagógicas. A falta de afinidade com o uso das ferramentas digitais foi apontada como fator que contribuiu para limitar a prática didática.

Nas falas dos participantes, evidenciou-se que a fragilidade da formação docente voltada para a prática pedagógica impacta na utilização de redes sociais como procedimento de ensino. Os participantes sinalizaram que os pontos frágeis do uso de tecnologia emergem de fatores ligados aos estudantes, à instituição de ensino e ao próprio docente. Neste contexto, destaca-se a dificuldade dos alunos relacionada ao acesso a recursos tecnológicos no ambiente extraclasse e a falta de habilidade do professor para lidar com a tecnologia e para associá-la ao conteúdo das disciplinas.

A codificação seletiva refinou todas as categorias e subcategorias emergindo a categoria central nominada "O desafio de envolver o aluno no processo de ensino aprendizagem e a distância entre geraçóes professor-aluno". Essa categoria permitiu integrar todas as outras categorias e subcategorias primitivas sem prejuízo semântico (Quadro 1).

\section{Discussão}

Nesse estudo o uso das TIC como recursos pedagógicos para o ensino da enfermagem se faz por meio das redes sociais (YouTube; Facebook e WhatsApp). No entanto, embora tenham sido apontados, os professores destacaram o desafio de envolver o aluno no 
Quadro 1. Distribuição das falas representativas que originaram as categorias, subcategorias e códigos da pesquisa com professores de enfermagem sobre dificuldades na utilização de tecnologia da informação no processo de ensino

\begin{tabular}{|c|c|c|c|c|}
\hline \multirow{5}{*}{ 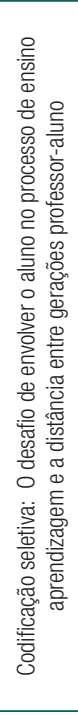 } & Categorias & Subcategorias & Códigos & Falas representativas \\
\hline & \multirow{4}{*}{$\begin{array}{l}\text { Fragilidade } \\
\text { da formação } \\
\text { docente }\end{array}$} & \multirow{3}{*}{ Conhecimento } & Aprendizagem & $\begin{array}{l}\text { A dificuldade da tecnologia... Olha, isso depende muito do conhecimento e da vivência do acadêmico. Nós temos acadêmicos } \\
\text { que têm mais acesso à tecnologia, como temos acadêmicos que quase não tem acesso a essa tecnologia, né? E quando eu, } \\
\text { como professor, sinto essa dificuldade no aluno, e é algo que eu estarei trabalhando, eu ensino o aluno a desenvolver essa } \\
\text { prática. (P1) } \\
\text { Esse é um momento desafiador para o docente, porque hoje trabalhamos com a geração Y, uma geração que fica antenada e } \\
\text { sintonizada em várias janelas. Eu penso no que eu utilizo durante o processo dialógico... Às vezes, você busca informação } \\
\text { rápido e o aluno está desatento e você faz uma pergunta... } 0 \text { desafio é buscar, naquele momento, a evidência que você está } \\
\text { discutindo. (P19) }\end{array}$ \\
\hline & & & Clínicas & $\begin{array}{l}0 \text { uso de celular e a tecnologia pode atrapalhar a prática pedagógica. Muito, mesmo! A primeira coisa que peço ao ir a prática } \\
\text { hospitalar é permanecer com os celulares desligados. (P11) }\end{array}$ \\
\hline & & & Associação & $\begin{array}{l}\text { No caso, ela (tecnologia de informação e comunicação) poderia ser complementar para implementação e ensino de algum } \\
\text { conteúdo. (P18) } \\
\text { Então, penso que temos que desenvolver tecnologia da informação o aluno está o tempo todo trabalhando determinado assunto. } \\
\text { E o aluno está o tempo todo pesquisando no celular se aquilo que falamos está certo. Então, temos que usar a tecnologia em } \\
\text { nosso favor em sala de aula, é claro que eu permito ele usar o celular ou tablet, mas eu tenho que ter controle se naquele } \\
\text { momento que ele está usando está relacionado ao contexto de sala de aula ou não. (P17) }\end{array}$ \\
\hline & & $\begin{array}{l}\text { Qualificação } \\
\text { permanente }\end{array}$ & $\begin{array}{l}\text { Educação } \\
\text { permanente }\end{array}$ & $\begin{array}{l}\text { Nós precisamos urgentemente realizar oficinas docentes em que possamos nos instrumentalizar para utilizar melhor essas } \\
\text { tecnologias novas. (P18) } \\
\text { A dificuldade que temos, muitas vezes é da motivação do servidor... para se adaptarem às novas metodologias e métodos de } \\
\text { trabalho. (P15) } \\
\text { Hoje, trabalhar com a docência requer uma formação contínua nossa. Principalmente do docente, aliás. Você tem que sempre } \\
\text { estar se atualizando. (P11) }\end{array}$ \\
\hline \multirow{5}{*}{ 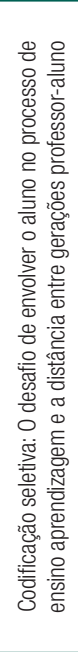 } & Categorias & Subcategorias & Códigos & Falas representativas \\
\hline & \begin{tabular}{|l|} 
Fragilidade \\
da formação \\
docente
\end{tabular} & $\begin{array}{l}\text { Qualificação } \\
\text { permanente }\end{array}$ & Didática & [...] eles chegam preparados e falta, para nós, saber como utilizar melhor a tecnologia a favor do ensino. (P18) \\
\hline & \multirow{3}{*}{$\begin{array}{l}\text { Utilização } \\
\text { de redes } \\
\text { sociais como } \\
\text { procedimento } \\
\text { de ensino }\end{array}$} & \multirow[t]{3}{*}{ Ferramentas } & WhatsApp & $\begin{array}{l}\text { Não acho que WhatsApp tem que fazer parte do contexto de sala de aula. Porque eu vejo que o processo de formação se } \\
\text { constitui de processos distintos. (P17) } \\
\text { Para mim isso é fabuloso! Mas precisamos saber usar isso corretamente, [...] porque de } 10 \text { em } 10 \text { minutos a gente olhar } \\
\text { WhatsApp, não é legal! (P16) } \\
{[\ldots . . .] \text { as redes sociais estão sendo mais interessantes que a rede informal da plataforma Moodle. Grupos do WhatsApp.... }} \\
\text { Orientações de pequenos grupos também têm acontecido. Então, hoje, essas novas tecnologias têm ajudado bastante! (P21) } \\
{[\ldots . .] \text { esse negócio de WhatsApp... desconcerta o professor! (P22) }} \\
{[\ldots . .] \text { temos o grupo no WhatsApp [...] e ele pode facilitar ou dificultar. Então, mostro para o aluno que, se ele tiver muito }} \\
\text { tempo no celular, vai perder tempo só respondendo a grupos!... Ele vai perder o que tem de interessante. Ele poderia estar lendo } \\
\text { artigos, estudando, pegando textos na biblioteca ou consultando outros livros... (P15) }\end{array}$ \\
\hline & & & Facebook & $\begin{array}{l}\text { A gente tem usado... Tem sido um aliado, porque, inicialmente, a universidade oferece a plataforma Moodle. Então, fomos } \\
\text { percebendo que as pessoas não acessavam e não avançavam. Então, decidimos criar um grupo fechado no Facebook e abolir, } \\
\text { por um semestre, o Moodle. E percebemos que até a atividade mais dificil para eles, quando é pelo Facebook, eles até curtem } \\
\text { [risos]! A adoção da rede social para nós, como o Facebook, tem sido muito interessante! (P21) }\end{array}$ \\
\hline & & & Youtube & $\begin{array}{l}\text { [...] Então percebemos que está sendo mais dinâmico usar tecnologia de informação e comunicação e mostrar as informações } \\
\text { ruins e boas. Teremos uma aula essa semana sobre temas importantes para a política na saúde e um aluno me mandou o que } \\
\text { achou no Youtube... (P17) }\end{array}$ \\
\hline
\end{tabular}

processo de ensino aprendizagem e a distância entre geraçôes professor-aluno foram elencados como dificultadores da aplicação das TIC em sala de aula. Outro aspecto apontado pelos participantes foi a falta de conhecimento sobre o uso das TIC durante sua formação docente, destacando a necessidade de qualificação permanente para uso das tecnologias.

A tecnologia desempenha papel relevante para tornar os estudantes ativos no seu processo de aprendizado possibilitando diversidade metodológica e educação centrada no estudante. $\mathrm{O}$ empoderamento dos futuros profissionais de enfermagem no uso de tecnologias nas suas atividades profissionais e para sua contínua capacitação requer a compreensão de que o professor é seu guia nesse processo. ${ }^{(20)}$
Assim, é impossível pensar em uso de TIC efetivo sem considerar a sua integraçáo no currículo dos graduandos e professores.

O Facebook é a rede social que lidera o ranking de utilização de TIC na assistência, na pesquisa e no ensino na enfermagem, seguido do Twitter e Whatsapp. ${ }^{(11,15)}$ No presente estudo, identificamos também o uso promissor do YouTube, plataforma pública de vídeos sobre temas distintos que pode agregar opção as ferramentas docentes. $\mathrm{O}$ uso de TIC para aproximar os conteúdos curriculares da realidade do aluno favorece formação crítica, reflexiva e generalista. Porém, nota-se resistência na utilização de estratégias diversificadas que sintonizam ferramentas online aos conteúdos ministrados. ${ }^{(16)}$ 
A atividade de enfermagem está imbricada a ação de educar, seja no contexto assistencial, por meio das atividades de educação em saúde, ou na formação de membros da equipe de enfermagem em instituiçóes de ensino e programas de educação continuada/permanente. Tal natureza tem sido confundida com competência adquirida de forma inata e esse pressuposto induz à errônea percepção de que o enfermeiro não precisa ser formado para práticas pedagógicas. ${ }^{(17)}$

Nesse contexto, a ampliação do uso das TIC está atrelada à capacidade de aplicá-las no processo de ensino. ${ }^{(18)}$ No entanto, a integração das TIC ao currículo de forma efetiva envolve também o experiência prévia de tecnologia no ensino, política educacional e desenvolvimento do conhecimento tecnológico pedagógico. Esse último compreende o sinergismo entre conteúdo proposto pelo projeto pedagógico do curso, metodologias de ensino-aprendizagem e recursos digitais. ${ }^{(19)}$ Esses dados podem explicar porque no nosso estudo os professores que relataram dificuldades na aplicação das TIC também referiram precisar de capacitação e apoio institucional para poder utilizá-las.

Os reflexos da incipiente formação dos enfermeiros na perspectiva das competências pedagógicas podem ser percebidos pela resistência e dificuldade do docente em implementar metodologias diferentes das práticas centradas no professor. Esse fato pode restringir as ferramentas utilizadas no processo de ensino, entre essas, as TIC. ${ }^{(21)}$

$\mathrm{Na}$ atualidade, as instituiçóes de ensino têm investido no uso de plataformas virtuais, que permitem a interaçáo professor- aluno. Entretanto, o que se tem percebido é que a utilização dessas plataformas tem se limitado a depósito de materiais, muito distante de práticas educativas inovadoras o que pode comprometer tanto o ensino híbrido quanto à distância. ${ }^{(9)} \mathrm{O}$ ensino à distância, em especial, tem crescido nos últimos anos e representa possibilidade de democratização e inclusáo, devido sua flexibilidade de acesso, custo e tempo. No entanto, ainda são incipientes ferramentas de avaliação da qualidade do ensino oferecido e persiste indefinição de conteúdos que podem ser trabalhados nessa modalidade em profissóes práticas, como a enfermagem. ${ }^{(22)}$

É preciso garantir que o conteúdo, os recursos e o suporte técnico necessário não representem barreiras ao uso da TIC como forma de ensino. ${ }^{(23)}$ Estima- se que poucas pessoas não possuam acesso à internet, celular e computador em diferentes países do mundo. Mesmo assim, essas pessoas, geralmente com renda mais baixa do que as demais, não podem deixar de ser consideradas quando se planeja o uso de TIC no ensino. ${ }^{(24)}$ As experiências dos docentes entrevistados no nosso estudo evidenciou que esse é um desafio para os professores inseridos nas salas de aula: unir a inovação e a perspectiva inclusiva no processo do ensino.

Além da questão do acesso do aluno a tecnologia, outro entrave ao uso das TIC, é a distância entre geraçóes professor-aluno. Isto, porque, na área da saúde pode ser composta por três geraçôes, denominadas X, $\mathrm{Y}$ e Z . Fazem parte da geração $\mathrm{X}$ as pessoas nascidas entre 1962 e 1980, os que nasceram entre 1981 e 1989 são chamados de geração $Y$, enquanto os nascidos a partir de 2000 constituem a geração Z. ${ }^{(25)}$ Por isso, o conflito de geraçôes pode ter sido dilema comum na prática dos docentes entrevistados no nosso estudo, onde quase a metade possuía 45 anos ou mais (ou seja, pertencentes a geração X) e os graduandos, por outro lado, tem se inserido cada vez mais jovens no ensino superior.

A operacionalização das TIC para a geração $X$ constitui desvantagem e limitação esperada, pois as geraçôes mais jovens já iniciam sua formação no contexto das TIC. Assim, o avanço tecnológico evidencia a necessidade de qualificação permanente para minimizar o sentimento de impotência desses professores e o conflito de geraçóes na prática pedagógica. ${ }^{(26)}$

A complexidade de trabalhar as TIC em saúde deve ser rotineiramente discutida sob a ótica de novas formas de aprendizagem que emergem dos processos educativos, os quais objetivam a formação de profissional comprometido com qualidade para desenvolver a ação profissional com desvelo e competência. Portanto, mudanças significativas devem ser uma constante na docência e não estão dissociadas da geração à qual o professor pertença ou esteja inserido. ${ }^{(26,27)}$

\section{Conclusão}

Nesse estudo, a utilização das TIC no processo de ensino e aprendizagem em enfermagem se fez por meio 
das redes sociais (YouTube; Facebook e WhatsApp). No entanto, embora venham sendo utilizadas, os professores relataram que o seu conhecimento no manuseio dessas tecnologias impacta no uso seu como procedimento de ensino. E, essa dificuldade é mais acentuada à medida em que aumenta a diferença de idade/geração entre estudantes e professores. Como perspectivas os resultados desse estudo têm potenciais benefícios para três áreas distintas: no ensino, por permitir a reflexão sobre a formação docente, a fim de alinhar a prática pedagógica ao perfil cada vez mais tecnológico dos acadêmicos, subsidiar o avanço dos cursos de enfermagem e favorecer a superação de barreiras ao uso das TIC no campo teórico-prático. $\mathrm{Na}$ pesquisa, fornece um escopo de conhecimento sobre dificuldades ao uso das TIC e fomenta a realização de novos estudos nessa área que representa o presente e o futuro do ensino. No sistema de saúde, a utilização das TIC pode favorecer formação de profissionais críticos e conectados as necessidades da população potencializando a qualidade do cuidado.

\section{Agradecimentos}

Os autores agradecem a FAPEG - Fundação de Amparo à Pesquisa do Estado de Goiás pela Bolsa de mestrado fornecida a mestranda Ângela Gilda Alves.

\section{Colaborações}

Alves AG, Cesar FCR, Martins CA, Ribeiro LCM, Oliveira LMAC, Barbosa MA e Moraes KL colaboraram com a concepção estudo, análise e interpretação dos dados, revisão crítica relevante do conteúdo intelectual e aprovação da versão final a ser publicada.

\section{Referências}

1. Brixey JJ, Newbold SK. Nursing Informatics Pioneers Embrace Social Media. Stud Health Technol Inform. 2017;245(1):1297.

2. Rouleau G, Gagnon MP, Côté J, Payne-Gagnon J, Hudson E, Dubois CA. Impact of information and communication technologies on nursing care: results of an overview of systematic reviews. J Med Internet Res. 2017;19(4):e122.
3. Hübner U, Shaw T, Thye J, Egbert N, Marin HF, Chang P, O'Connor S, Day K, Honey M, Blake R, Hovenga E, Skiba D, Ball MJ. Technology Informatics Guiding Education Reform - TIGER. Methods Inf Med. 2018 Jun;57(S 01):e30-e42.

4. O'Connor S, Hubner U, Shaw T, Blake R, Ball M. Time for TIGER to ROAR! Technology Informatics Guiding Education Reform. Nurse Educ Today. 2017;58(1):78-81.

5. Tubaishat A, Aljezawi M, Al-Rawajfah OM, Habiballah L, Akhu-Zaheya LM. Exploring changes in nursing students' attitudes towards the use of technology: A four-wave longitudinal panel study. Nurse Educ Today. 2016 Mar;38(5):101-6.

6. Mantas J, Hasman A. IMIA Educational Recommendations and Nursing Informatics. Stud Health Technol Inform. 2017;232(1):20-30.

7. Fagerström C, Tuvesson H, Axelsson L, Nilsson L. The role of ICT in nursing practice: an integrative literature review of the Swedish context. Scand J Caring Sci. 2017 ;31(3):434-48.

8. Risling T. Educating the nurses of 2025: technology trends of the next decade. Nurse Educ Pract. 2017 Jan;22(1):89-92.

9. Raman J. Mobile technology in nursing education: where do we go from here? A review of the literature. Nurse Educ Today. 2015 May;35(5):663-72.

10. Chiou SF, Su HC, Huang EW. The Application of Information and Communication Technology (ICT) in Nursing Education]. Hu Li Za Zhi. 2017 Dec;64(6):5-11. Chinese.

11. Mesquita AC, Zamarioli CM, Fulquini FL, Carvalho EC, Angerami EL. Social networks in nursing work processes: an integrative literature review. Rev Esc Enferm USP. 2017;51(1):e03219.

12. Vygotsky LS. A formação social da mente. São Paulo: Martins Fontes; 1994.

13. Corbin JM, Strauss A. Grounded theory research: Procedures, canons, and evaluative criteria. Qual Sociol. 1990;13(1):3-21.

14. Strauss A, Corbin J. Basics of qualitative research: grounded theory procedures and techniques. 2nd ed. Newbury Park: Sage; 1998.

15. Kakushi LE, Évora YD. Social networking in nursing education: integrative literature review. Rev Lat Am Enfermagem; 2016; 24:e2409.

16. Guerra FF, Balbino CA, Moreira AM. Tecnologias e currículo: impactos na prática educativa e no papel do professor. Plures Humanidades; 2017;18(1):75-93.

17. Silva MV, Carvalho Filha FS, Nascimento FL, Branco TB, Lima $\mathrm{ND}$, Miranda RH. A dicotomia entre teoria e prática na formação do enfermeiro docente. Revista Recien - Rev Cient Enferm. 2018;8(22):93.

18. Gessinger RM, Moraes MC, Lopes Leite L, Valderez Lima MR. 0 uso pedagógico de recursos tecnológicos como estratégia para qualificar 0 ensino e contribuir para a redução da evasão na educação superior. CLABES. 2016;1(1):1-7.

19. Chen HJ, Liao LL, Chang YC, Hung CC, Chang LC. Factors Influencing Technology Integration in the Curriculum for Taiwanese Health Profession Educators: A Mixed-Methods Study. Int J Environ Res Public Health. 2019;16(14):1-16.

20. Uslu 0. Factors associated with technology integration to improve instructional abilities: A path model. Aust J Teach Educ. 2018;43(4):3142.

21. Mesquita SK, Meneses RM, Ramos DK. Metodologias ativas de ensino/ aprendizagem: dificuldades de docentes de um curso de enfermagem. Trab Educ Saúde. 2016;14(2):473-86. 
22. Tanaka EZ, Sartori DV, Ferreira LR, Bermejo LJ. A educação a distância nos cursos de graduação em enfermagem: aplicação e efetividade. RPGE. 2017;21(1):831-41.

23. O'Connor S, Andrews T. Smartphones and mobile applications (apps) in clinical nursing education: A student perspective. Nurse Educ Today. 2018;69(1):172-8.

24. Knop MF. Exclusão digital, diferenças no acesso e uso de tecnologias de informação e comunicação: questões conceituais, metodológicas e empíricas. CADECS. 2018;5(2):39-58.
25. Levickaite R. Generations $x, y, z$ : how social networks form the concept of the world without borders (the case of Lithuania). LIMES: Cultural Regionalistics. 2010;3(2):170-83.

26. Fernández-Alemán JL, Sánchez García AB, López Montesinos MJ, Marqués-Sánchez P, Bayón Darkistade E, Pérez Rivera FJ. Exploring the Use of information and communication technologies and social networks among university nursing faculty staff. An opinion survey. Invest Educ Enferm. 2014;32(3):438-50.

27. Mather C, Cummings E, Gale F. Mobile Learning in Nursing: tales from the Profession. Stud Health Technol Inform. 2018;252(1):112-7. 